\title{
ACCOUNTING FOR TIME DEPENDENCE IN LARGE-SCALE MULTIPLE TESTING OF EVENT-RELATED POTENTIAL DATA: ONLINE SUPPLEMENT
}

\author{
By Ching-Fan Sheu * Émeline Perthame ${ }^{\dagger}$, Yuh-Shiow Lee ${ }^{\ddagger}$ \\ , AND David Causeur ${ }^{\dagger}$ \\ National Cheng Kung University, Tainan, Taiwan * and \\ Agrocampus-Ouest, Rennes, France ${ }^{\dagger}$ and National Chung Cheng \\ University, Chiayi, Taiwan $\ddagger$
}

\begin{abstract}
Event-related potentials (ERPs) are recordings of electrical activity along the scalp time-locked to perceptual, motor and cognitive events. Because ERP signals are often rare and weak, relative to the large between-subject variability, establishing significant associations between ERPs and behavioral (or experimental) variables of interest poses major challenges for statistical analysis.

Noting that ERP time dependence exhibits a block pattern suggesting strong local and long-range autocorrelation components, we propose a flexible factor modeling of dependence. An adaptive factor adjustment procedure is derived from a joint estimation of the signal and noise processes, given a prior knowledge of the noise-alone intervals. A simulation study is presented using known signals embedded in a real dependence structure extracted from authentic ERP measurements. The proposed procedure performs well compared with existing multiple testing procedures and is more powerful at discovering interesting ERP features.
\end{abstract}

1. The impact of ERP time-dependence on multiple testing results. To demonstrate the impact of time dependence on the ability of multiple testing procedures to identify a predetermined true signal, we conduct a simulation study in which $n \times T$ ERP data are generated according to model (3.1) in the main document, with $n=20$ and $T=1,000$ matching the number of participants and time frames of the directed forgetting experiment introduced in Section 2 of the main document. For each simulation run, the only covariate $x$ is the centered recognition performance as observed in the TBR condition of the experiment. We set $\mu_{t}=0, b_{t}=0$, for all $t=1, \ldots, T$, and use the sample estimates from the observed ERP curves at electrode $\mathrm{CZ}$ for the residual standard deviations $\sigma_{t}, t=1, \ldots, T$.

AMS 2000 subject classifications: Primary 62H25, 62H30, 62J12, 62J07

Keywords and phrases: Dependence, ERP data, High-dimensional data, Multiple testing

imsart-aoas ver. 2013/03/06 file: AOAS1407021R2-SUPPA.tex date: October 20, 2015 
Two correlation matrices are considered for the residual errors: an independent structure, $I_{T}$, or the 5 -factor model displayed in Figure 3 of the main document. The true signals $t \mapsto \beta_{t}$ have the same bell shape on a same support from $450 \mathrm{~ms}$ to $550 \mathrm{~ms}$ with varying peak heights starting at zero, and then from 1.5 to 12.5 in equal step sizes of 0.1 . Figure 1 shows the corresponding powers of the individual t-tests of $H_{0, t}: \beta_{t}=0$ for a Type-I error rate $\alpha=0.05$. For each of the possible combinations of a correlation matrix and a signal amplitude, 1,000 ERP datasets are generated. This simulation study compares the GB procedure [?] with a graphical threshold of 0.05 and two FDR-controlling procedures: BH [?] and BY [?] with FDR control level at $\alpha=0.05$.

For each simulation run, the procedures are assessed by the FDR, the Positive Predictive Value (PPV), also called precision, defined as the expected proportion of correct rejections of the null among the positives and the probability of no rejection (PNR), defined as the expected proportion of datasets for which no null was rejected. Figure 2 summarizes the results.

Moreover, in the present biomedical signal processing issue, let us think of a multiple testing procedure as a tool to detect time intervals on which a signal is nonzero. In this context, sensitivity and resolution are common ways to compare the accuracy of measurement tools. Sensitivity refers here to the lowest signal amplitude for which the method starts detecting weak impulses. In the present multiple testing situation, we therefore propose to define the sensitivity of a multiple testing procedure as the minimum peak height for which 1-PNR exceeds 0.1:

$$
\text { Sensitivity }=\min \left\{\max _{t} \beta_{t}, 1-\mathrm{PNR} \geq 0.10\right\} .
$$

Similarly, the resolution of a multiple testing procedure is the extent to which it can detect the time interval of nonzero signal. We propose to define the resolution of a method as the minimum peak height for which PPV exceeds 0.9 :

$$
\text { Resolution }=\min \left\{\max _{t} \beta_{t}, \mathrm{PPV} \geq 0.90\right\} .
$$

The sensitivity and resolution of the 3 multiple testing procedures compared in the present simulation study are reported in Table 1.

First, as expected, in both cases of independent and dependent noise, the BY [?] and BH [?] procedures control the FDR. Indeed, both methods control the FDR at a lower level than required (here 0.05) under dependence, especially BY, inducing a marked loss of precision relative to $\mathrm{BH}$. This finding is consistent with those reported in many previous comparative

imsart-aoas ver. 2013/03/06 file: AOAS1407021R2-SUPPA.tex date: October 20, 2015 


\section{True power in simulation study}

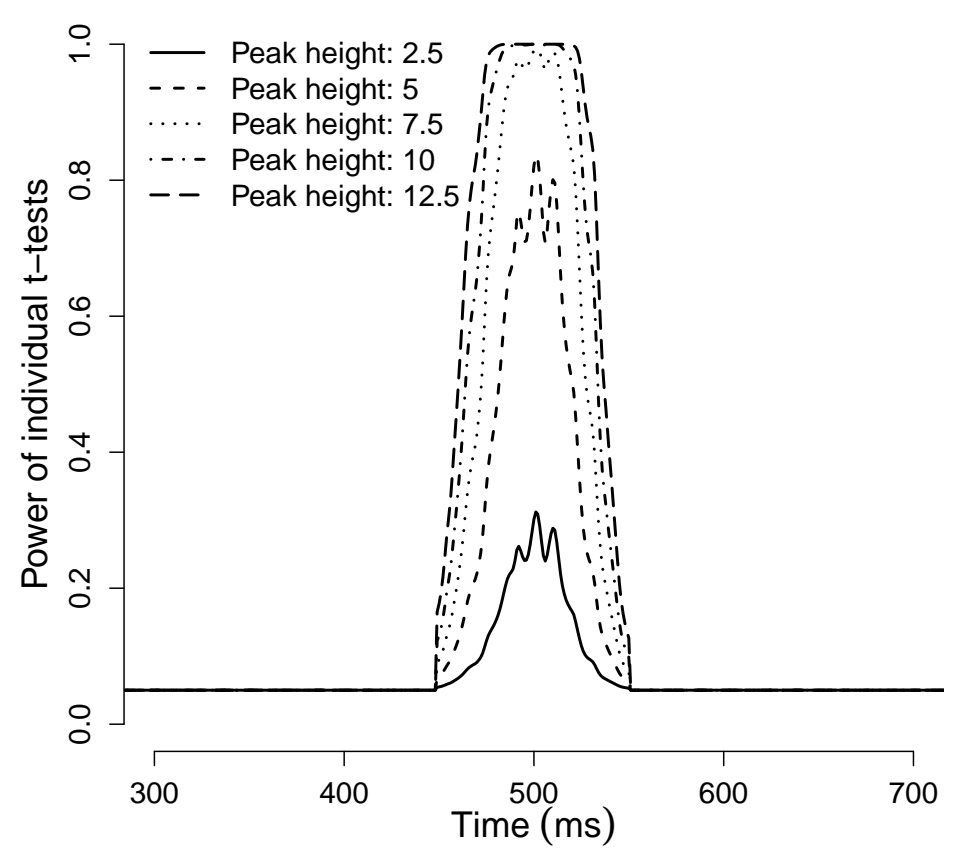

Fig 1: True powers of individual t-tests for $H_{0, t}: \beta_{t}=0$ in the simulation study. Signals have the same shape over the interval [450 ms, $550 \mathrm{~ms}$ ] with varying peak heights from 0 to 12.5 : only power curves with peak heights at $2.5,5.0,7.5,10.0$ and 12.5 are displayed here.

studies of large-scale significance analysis methods [?]. Table 1 confirms that dependence tends to reduce the sensitivity of $\mathrm{BH}$ and $\mathrm{BY}$.

On the contrary, GB does not ensure the required FDR level under dependence, especially when the signal is weak to moderate, while on the contrary it seems to overcontrol the FDR under independence. It can also be deduced from Table 1 that the sensitivity of GB is increased by dependence. Indeed, in the dependent case, even when the true signal is zero on the whole time frame, the probability that GB detects significant time points exceeds 0.10 . These findings are also consistent with those reported by ? in which data were generated according to a model of ERP [?] embedded with a first-order autoregressive noise process.

Generally, the three procedures turn out to be less precise under depen-

imsart-aoas ver. 2013/03/06 file: AOAS1407021R2-SUPPA.tex date: October 20, 2015 

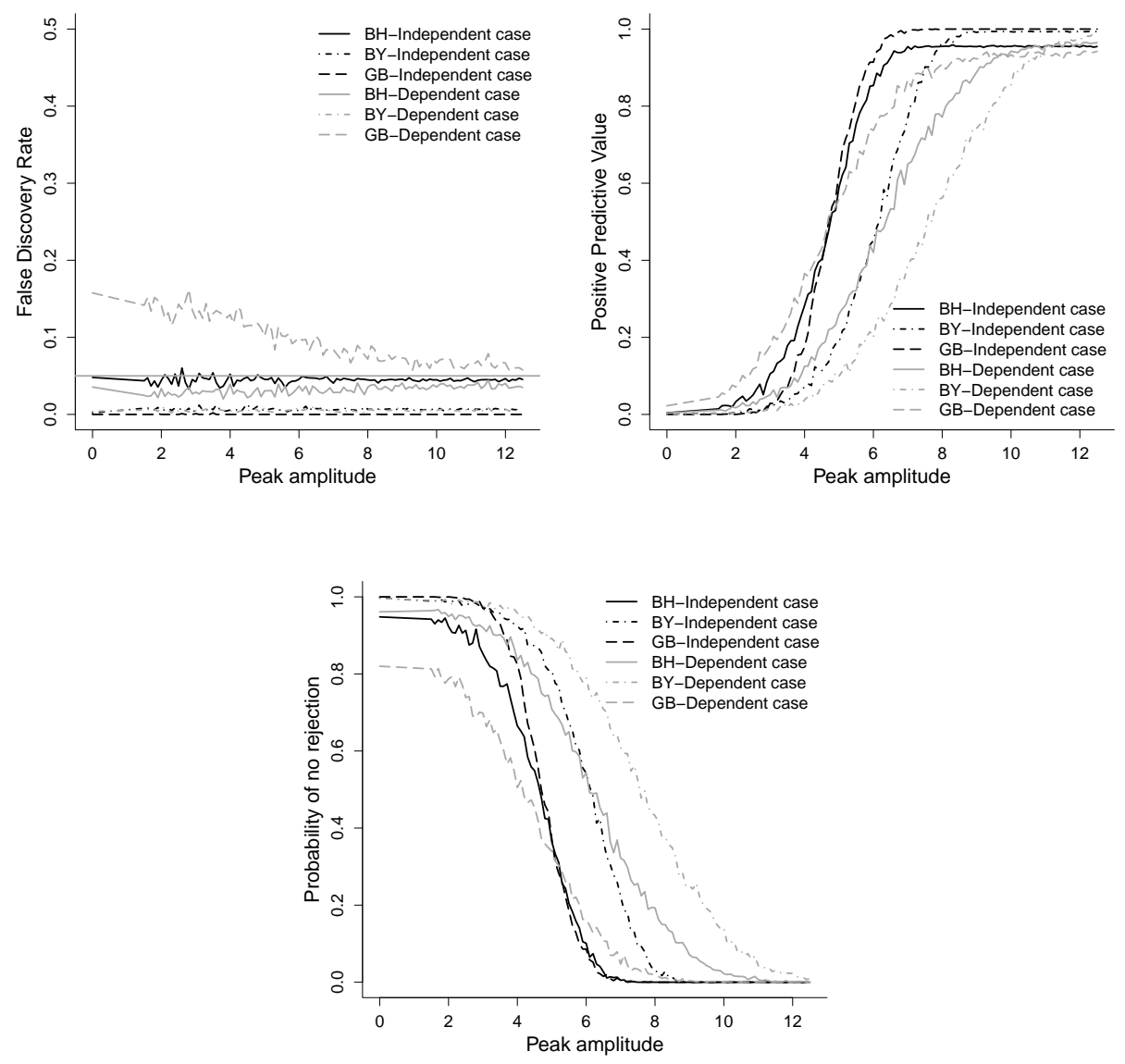

Fig 2: False Discovery Rate (top left), Positive Predictive Value (top right), Probability of No Rejection (bottom ) for the comparative study of 3 multiple testing procedures (solid curves for BH, dotted and dashed curves for BY and dashed curves for GB) using simulations of ERP data with independent (black) and dependent (dark gray) noise. For each peak amplitude $\max _{t} \beta_{t}$, 1,000 datasets were generated.

dence than under independence. Consistently, it is deduced from Table 1 that dependence lowers markedly the resolution of BH, BY and GB. However, GB shows a better ability to find out the peak, in terms of PPV, under dependence and, provided the signal amplitude is not too small, also under independence. 
Moreover, dependence also seems to affect the stability of signal detection using $\mathrm{BH}$ and BY. Indeed, the probability of no rejection (PNR) decreases much more slowly along with the peak height when the tests are highly correlated for all the procedures. Recall that the FDR and the PNR are related by: $\mathrm{FDR}=\mathrm{pFDR}(1-\mathrm{PNR})$, where $\mathrm{pFDR}=\mathbb{E}(\mathrm{FDP} \mid \mathrm{R}>0)$ is called the positive FDR [?]. Though, in balance, dependence does not seem to affect the overall level of FDR control, it tends to increase the PNR as well as the pFDR. In other words, for highly dependent test statistics, the chance of declaring at least one time point as significant is much lower than that for independent statistics; moreover, when positives are found, the expected false discovery proportion is much larger.

TABLE 1

Sensitivity and resolution (see definitions (1.1) and (1.2)) of 3 multiple testing procedures (BH, BY and $G B)$ using simulations of ERP data with independent and dependent noise. For $B H$ and $B Y$, the FDR control level is 0.05. The graphical threshold in $G B$ is set to 0.05 .

\begin{tabular}{l|cc|cc}
\hline \multirow{2}{*}{ Method } & \multicolumn{2}{|c|}{ Independent noise } & \multicolumn{2}{c}{ Dependent noise } \\
& Sensitivity & Resolution & Sensitivity & Resolution \\
\hline BH & 2.6 & 6.3 & 3.6 & 9.1 \\
BY & 4.3 & 7.5 & 4.9 & 10.4 \\
GB & 3.7 & 5.9 & 0.0 & 7.9 \\
\hline
\end{tabular}

\section{References.}

Benjamini, Y. and Hochberg, Y. (1995). Controlling the False Discovery Rate: a practical and powerful approach to multiple testing. Journal of the Royal Statistical Society, Series B $\mathbf{5 7}$ 289-300.

Benjamini, Y. and Yekutieli, D. (2001). The control of the False Discovery Rate in multiple testing under dependency. Annals of Statistics 29 1165-1188.

Causeur, D., Chu, M. C., Hsieh, S. and Sheu, C. F. (2012). A factor-adjusted multiple testing procedure for ERP data analysis. Behavior Research Methods 44 635-643.

Friguet, C., Kloareg, M. and Causeur, D. (2009). A factor model approach to multiple testing under dependence. Journal of the American Statistical Association 104 1406-1415.

Guthrie, D. and Buchwald, J. S. (1991). Significance testing of difference potentials. Psychophysiology 28 240-244.

Storey, J. D. and Tibshirani, R. (2003). Statistical significance for genome-wide experiments. Proceedings of the National Academy of Sciences of the United States of America 100 9440-9445.

Yeung, N., Bogacz, R., Holroyd, C. B. and Cohen, J. D. (2004). Detection of synchronized oscillations in the electroencephalogram: An evaluation of methods. Psychophysiology 41 822-832. 
Address of the First author INTITUTE OF EDUCATION

National Cheng Kung University

1 University Road, Tainan 701, Taiman.

E-MAIL: csheu@mail.ncku.edu.tw
Address of the Second and Fourth author Agrocampus Ouest, IRMAR UMR 6625 CNRS 65 RUE DE St-Brieuc, CS 84215

35042 Rennes Cedex, France.

E-MAIL: emeline.perthame@agrocampus-ouest.fr david.causeur@agrocampus-ouest.fr

AdDress OF THE Third AUTHOR

Department of PSychology

National Chung Cheng University

168 University Road, Chiayi 621, Taiwan.

E-MAIL: psyysl@ccu.edu.tw 